J3eA - Vol. 2 - 5 (2003).

DOI : 10.1051/bib-j3ea:2003005

\title{
Didacticiel multimédia : « Électronique des microsystèmes, l'apport de la technique des capacités commutées 》
}

\author{
F. Baillieu * ${ }^{*}$ et O. Français ** (ESIEE, Noisy-le-Grand) \\ Mis en ligne le 4 avril 2003.
}

\begin{abstract}
Résumé
Cet article présente un didacticiel dédié à l'électronique des microsystèmes dans le cadre de l'utilisation des capacités commutées et quelques éléments sur son mode d'emploi. Ce didacticiel est développé à l'aide du logiciel Flash 5 ; il propose à l'utilisateur des scénarios didactiques destinés à rendre l'apprentissage dynamique et attractif. Le didacticiel est renforcé par l'utilisation d'une maquette pédagogique qui permet d'associer la théorie à la pratique. Plusieurs TP ont été développés autour de cette maquette. Ce didacticiel a déjà eu un impact positif auprès des étudiants qui s'est traduit par de meilleurs résultats aux examens.
\end{abstract}

Mots-clés : didacticiel, Flash, microsystèmes, capacités commutées, maquette pédagogique.

(C) EDP Sciences, 2003.

\begin{abstract}
Niveau de connaissances requis. Composants, électronique des fonctions, physique générale.
Niveau des étudiants. Deuxième cycle (licence EEA, GEII, Bac+3), seconde année d'IUT en Génie électrique, première année d'école d'ingénieur. La spécificité du didacticiel permet d'approfondir le sujet et devient pour certaines parties d'un niveau Bac+5 (école d'ingénieur, DESS).
\end{abstract}

* Ingénieur EBP (1962) et ESE (1964), François Baillieu ${ }^{1}$ débute sa carrière dans l'industrie.

En 1967 il entre au Groupe ESIEE comme ingénieur responsable d'études en charge de la recherche et du suivi pédagogique de projets sous contrat réalisés par les étudiants. En 1975, professeur associé à l'origine de la Salle Blanche et du programme d'enseignement correspondant, il est nommé responsable du Département Physique et Micro-électronique, fonction qu'il exercera jusqu'en 1992. Visiting professor à l'Université de Stanford, il développe et réalise des amplificateurs opérationnels NMOS. Au retour des USA, à la demande du Ministère de l'Industrie, il rédige un rapport de synthèse sur les activités de la Silicon valley. En 1986, nommé chargé de mission pour l'électronique au Ministère de l'Enseignement supérieur et de la Recherche, il contribue durant six années au lancement d'actions institutionnelles concernant les capteurs et les microsystèmes.

Nommé Professeur du Groupe ESIEE en 1993, Directeur de la Recherche et de l'Appui aux Entreprises de 1997 à 1999, il participe ensuite à la création du Pôle Francilien pour les Microsystèmes, le PFM, avec pour objectif de fédérer les activités de R\&D de l'ESIEE, l'IEF (Université Paris-Sud), l'ENS-Cachan, du LURE,..., en matière de microsystèmes. Il en sera le Président de 1999 à 2001. Par ailleurs, il exerce une activité de recherche et d'enseignement dans le domaine des micro-technologies, tout particulièrement en électronique des microsystèmes et contribue à 31 publications et trois ouvrages.

À partir de 1999 son intérêt pour les Techniques de l'Information et de la Communication appliquées à l'Enseignement (TICE) conduit François Baillieu à substituer aux supports pédagogiques issus de la bureautique, des supports plus intéractifs. C'est dans cette perspective qu'il développe sous Flash 5, le cours intitulé Électronique des microsystèmes, l'apport de la technique des capacités commutées maintenant proposé en ligne. En outre, pour faciliter la matérialisation d'autres projets sur cette plateforme très prometteuse, il souhaite mettre à disposition des enseignants qui en feraient la demande des scénarios « source » incorporant des animations réutilisables et partager ainsi avec eux son expérience. 
François Baillieu est Chevalier dans l'ordre des Palmes Académiques, membre Sénior de la SEE et Professeur Honoraire du Groupe ESIEE.

a e-mail : f.baillieu@wanadoo.fr (auteur correspondant)

** Olivier Français ${ }^{1}$ est né à Paris (France) en 1970. Il a obtenu l'agrégation de Génie Electrique en 1993 puis un Doctorat de l'Ecole Normale Supérieure de Cachan en 1998 sur la modélisation et réalisation d'une micropompe électrostatique à membrane. Il a intégré le groupe ESIEE en 1998 au sein du laboratoire Électronique et Microélectronique en tant qu'enseignant-chercheur. Il a focalisé ses activités de recherche sur le domaine de la microfluidique et des micro-capteurs (MEMS) en s'appuyant sur les moyens en microtechnologie du Groupe ESIEE (Salle Blanche). Il développe actuellement une activité en direction des Bio-MEMS. Il est actuellement responsable de programmes d'enseignement sur les microsystèmes dans le cursus ingénieur du Groupe ESIEE.

e-mail : o.francais@esiee.fr

${ }^{1}$ Groupe ESIEE, Cité Descartes, B.P. 99, F-93162 Noisy-le-Grand, France.

\section{Objectifs de l'utilisation du didacticiel}

Ce didacticiel s'inscrit dans le cadre d'une réflexion pédagogique globale qui requiert le développement et l'appropriation d'outils modernes interactifs, permettant de donner à l'étudiant un enseignement à la fois théorique et pratique, consistant et accessible, avec plusieurs exemples d'application. Trois idées majeures sont à la base de son organisation :

- dire pourquoi avant de dire comment ;

- $\quad$ permettre des démarches pédagogiques variées où se mêlent des approches descendantes (du système vers le composant) et ascendantes (du composant vers le système), au moyen d'un navigateur ;

- réserver les possibilités d'impression pour faciliter la pratique d'un travail personnel sur papier : l'écriture reste l'outil privilégié de la concentration.

\section{Le didacticiel aujourd'hui}

Ce didacticiel, accessible en lecture seule à l'adresse : www.esiee.fr/ems, est développé par notre équipe sous Flash 5 qui est un logiciel de la société Macromedia adapté à la création d'animations accessibles par Internet ; les exécutables étant de petite taille. Le navigateur (Fig.1) donne accès à quatre tables des matières :

- composants et circuit CMOS [1] ;

- capacités commutées : les bases;

- capacités commutées approfondies ;

- l'électronique des microsystèmes [2,3]. 


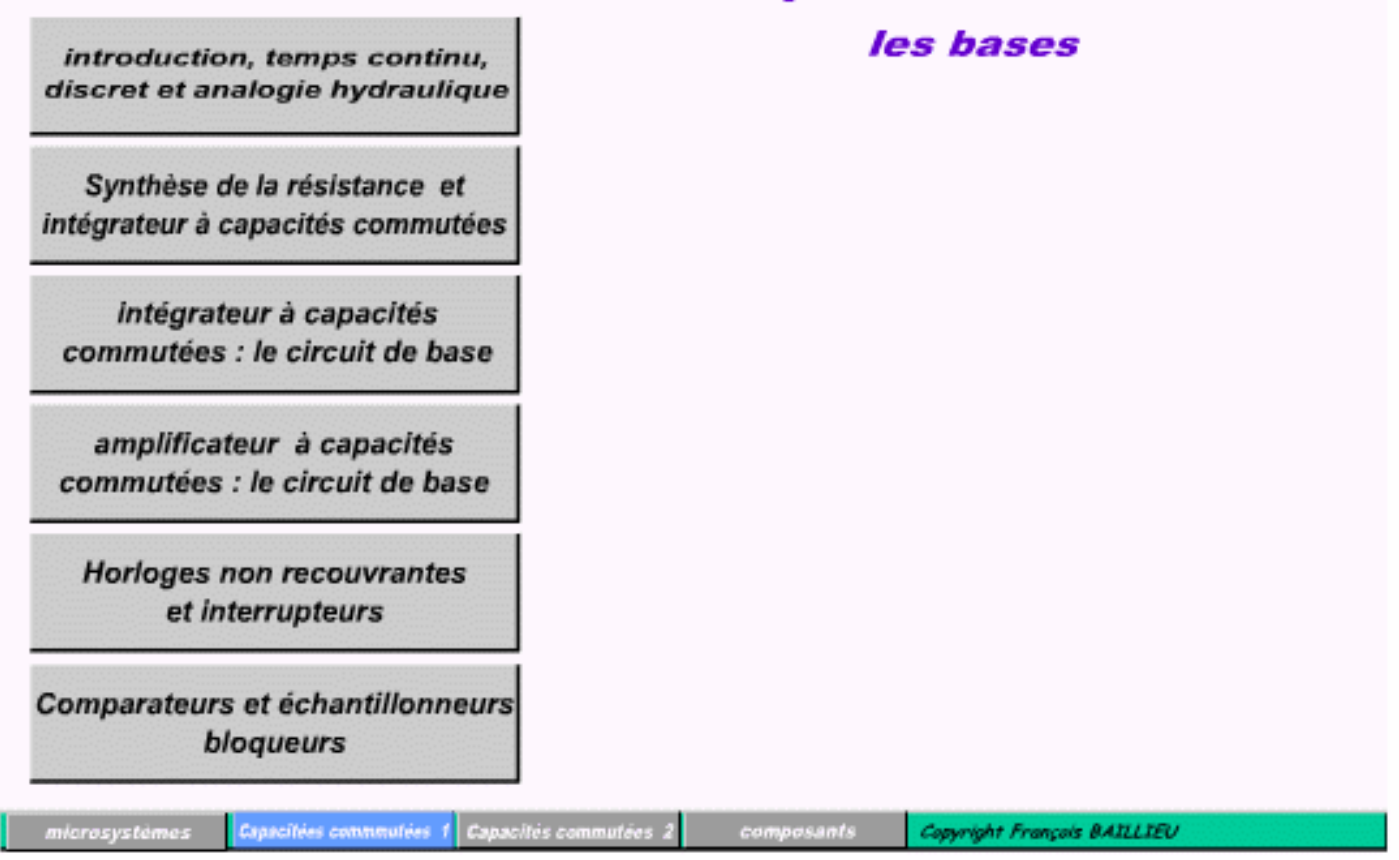

Fig. 1. Navigateur avec un exemple de table des matières.

Chaque table des matières contient plusieurs chapitres dont il est possible de prendre rapidement connaissance en glissant la souris sur les boutons correspondants avant de procéder à l'ouverture de l'un d'entre eux en « cliquant». L'exécutable de chaque chapitre n'excède pas $100 \mathrm{Ko}$ ce qui rend le temps d'accès par Internet tout à fait acceptable.

- Dans chaque chapitre, l'utilisateur peut à loisir naviguer dans les deux sens à l'intérieur d'un scénario à l'aide des boutons droite/gauche en bas d'image, ou passer d'un scénario à l'autre selon un plan logique préétabli, à l'aide des boutons avant/arrière ; à tout instant il peut revenir à la table des matières du chapitre étudié et au navigateur. Il dispose ainsi d'un véritable livre où toutes les figures sont des dessins animés dont le défilement est contrôlé de façon semi-automatique.

- Les scénarios exploitent souvent les analogies - hydrauliques et mécaniques - et les animations pour introduire simplement le principe d'une fonctionnalité électronique avant sa matérialisation sous la forme d'un schéma électrique.

- Les animations sont aussi particulièrement utiles pour mettre en évidence le mouvement des charges dans les circuits à capacités commutées et faciliter ainsi la compréhension de notions qui ne sont pas du tout familières aux étudiants qui débutent l'étude des systèmes analogiques à temps discret; citons à titre d'exemple :

○ la formation d'un système électrostatique isolé ;

- la redistribution des charges à l'intérieur d'un système électrostatique isolé ;

- le traitement de l'injection de charges par les horloges...;

- l'exploitation de la reconfigurabilité des circuits à temps discret pour réduire l'impact de certains défauts tels que l'offset, le bruit, etc., sur les performances.

\section{Notre expérience}

Dès le début de son développement, il y a quatre ans, le didacticiel a été mis à la disposition des étudiants. Par rapport à une approche plus classique précédemment en vigueur, ceci a eu pour conséquence une amélioration nette des résultats aux contrôles, validée sur une population très variée d'étudiants :

Ce didacticiel est en effet utilisé à l'ESIEE au niveau Ingénieur comme au niveau Technicien supérieur, dans le DESS de micro-électronique de l'Université Blaise Pascal, à l'ISEM. Il est exploitable de diverses façons : 
- $\quad$ en substitution des transparents ;

- $\quad$ en mise à disposition des étudiants par Internet ou l'Intranet local ;

- pour la post-assistance à distance.

Par rapport aux outils issus de la Bureautique comme Power Point, l'apport de la plateforme Flash 5 consiste en :

- une qualité didactique des animations bien supérieure ;

- une excellente portabilité et un accès rapide sur Internet (pour mémoire, les fichiers ainsi accessibles sont des fichiers $s w f$ qui sont de simples exécutables ne contenant aucune information sur la construction des scénarios inscrite dans les fichiers fla).

\section{Quelques éléments descriptifs}

Les figures 2, 3 et 4 présentent quelques copies d'image correspondant à des étapes d'animations illustrant bien mal leurs qualités didactiques.

Les scènes animées permettent de faciliter la compréhension des phénomènes. Ainsi, dans les animations illustrées par les figures 2 et 3, les notions de transfert de charges sont mises en valeur de manière dynamique de telle façon que l'étudiant visualise ce qui se passe. Tandis que dans l'animation illustrée par la figure 4, c'est le comportement de l'amplificateur à capacités commutées qui est expliqué... Pour se convaincre de l'intérêt de ces animations mieux vaut aller faire un tour à l'adresse : www.esiee.fr/ems.

\section{L'homologue hydraulique de l'intégrateur à capacités commutées}

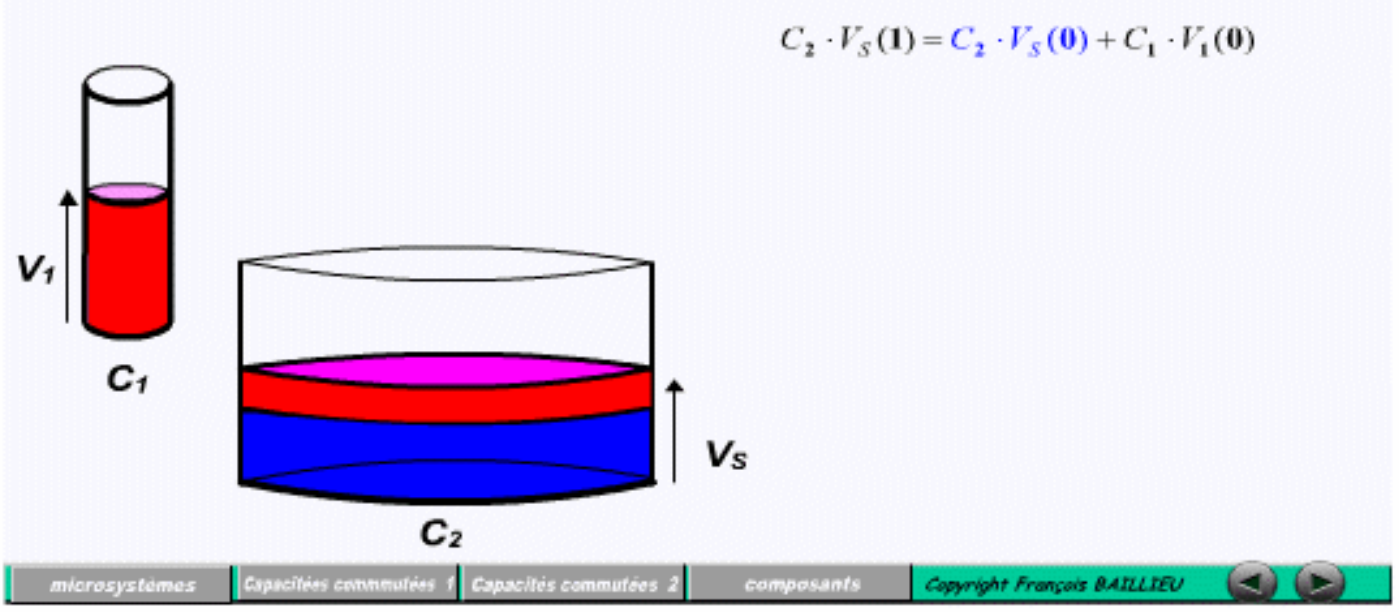

Fig. 2. L'analogie hydraulique de l'intégrateur. 


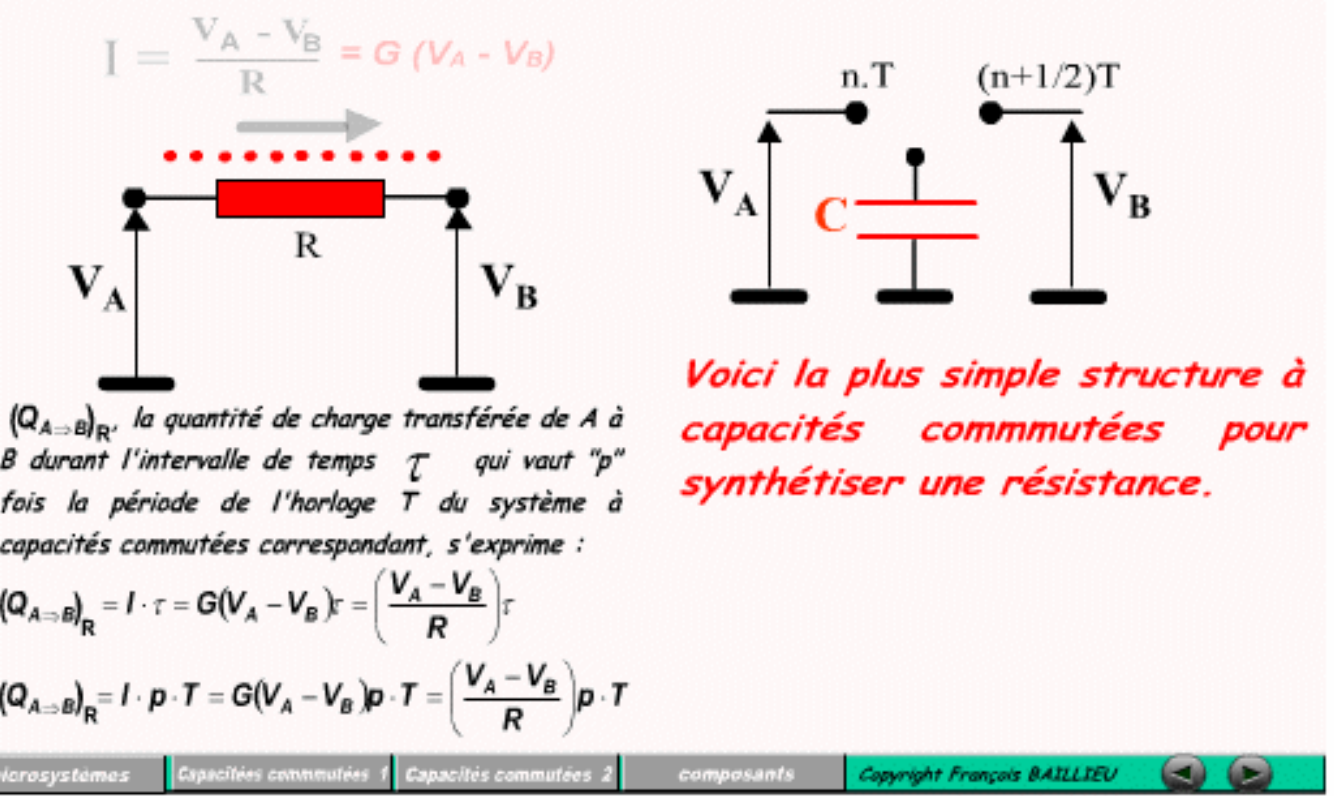

Fig. 3. Synthèse d'une résistance en capacités commutées.

\section{L'amplificateur à capacités commutées de base : Comment éliminer un commutateur? Voici la solution.

I) $|\mathrm{nT} ;(\mathrm{n}+1 / 2) \mathrm{T}|$

$$
\begin{aligned}
& \left(Q_{\mathrm{A}}\right)_{\mathrm{I}}=-\mathrm{C}_{1} \cdot \mathrm{V}_{\mathrm{I}}(\mathrm{nT}) \\
& \mathrm{V}_{\mathrm{S}}(\mathrm{nT})=0 \\
& \text { pré chargé et autozéro }
\end{aligned}
$$

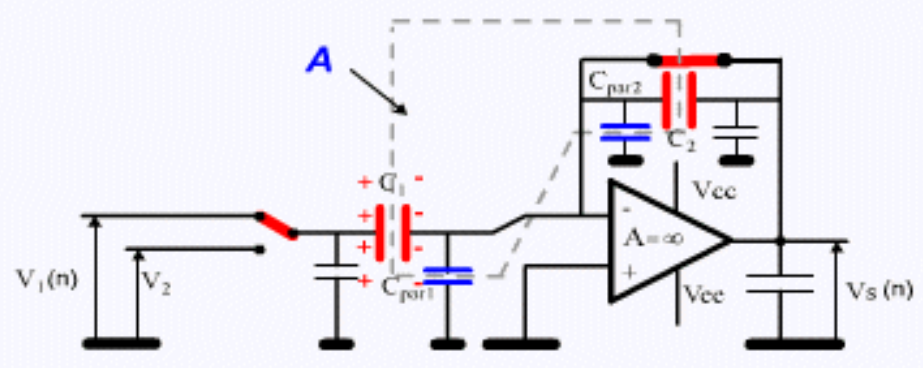

Fig. 4. Amplificateur à capacités commutées.

Un chemin pédagogique précis étant proposé à l'étudiant, il peut aborder progressivement les structures à étudier. En fonction de sa vitesse d'apprentissage, il peut ensuite choisir de façon autonome les parties du cours qu'il souhaite approfondir de manière interactive. 


\section{Travaux pratiques associés}

Deux séances de travaux pratiques ont été adjointes au didacticiel et s'appuient sur la réalisation d'un circuit imprimé permettant de mettre en valeur les points abordés lors de l'utilisation du didacticiel. À ce titre nous tenons à remercier Laurent Bues et Georges Bussignies qui ont développé la carte sous Mentor Graphics. Cette carte est constituée d'un modulateur Sigma Delta $(\Sigma \Delta)$ du premier ordre à capacités commutées, reconfigurable pour varier les modes de fonctionnement. En outre cette carte peut être pilotée par un micro contrôleur.

L'ensemble constitue alors un mésosystème intelligent qui préfigure l'architecture d'un véritable microsystème. Le découpage en deux séances de 4 heures est tel qu'il permet une découverte progressive d'un modulateur $\Sigma \Delta$.

Lors de la première séance, les étudiants découvrent l'intégrateur à capacités commutées commandé par des horloges non recouvrantes. Ils peuvent faire varier l'ensemble des paramètres afin d'assimiler le concept de transfert de charge entre deux condensateurs. Le principe de la synthèse d'une résistance par des capacités commutées est validé expérimentalement.

La deuxième séance s'appuie sur les résultats du premier TP et les applique à une chaîne d'acquisition de mesures utilisant le principe du modulateur sigma delta.

Le modulateur reprend l'intégrateur à capacités commutées qui est utilisé en rebouclage conditionnel à l'aide d'un comparateur. La première mesure consiste en la numérisation d'une humidité acquise par capteur capacitif. La seconde consiste en la numérisation d'une tension issue d'un pont diviseur incorporant un capteur de gaz résistif.

\section{Séance TP $\mathrm{N}^{\circ} 1$ :}

- horloges non recouvrantes ;

Séance TP N०2:

- intégrateur à capacités commutées.

- chaîne d'acquisition et de mesure à base de modulateur Sigma Delta ;

- mesure de capacité

- mesure de tension.

La structure de la carte est présentée figure 5. On y retrouve le modulateur Sigma Delta qui s'appuie sur un intégrateur à capacités commutées ainsi qu'une horloge non recouvrante utilisant un NE555 comme horloge de référence (le temps de non-recouvrement peut être modifié par l'utilisation de capacité externe Creg1 et Creg2) et un comparateur réalisé avec une bascule 4013.

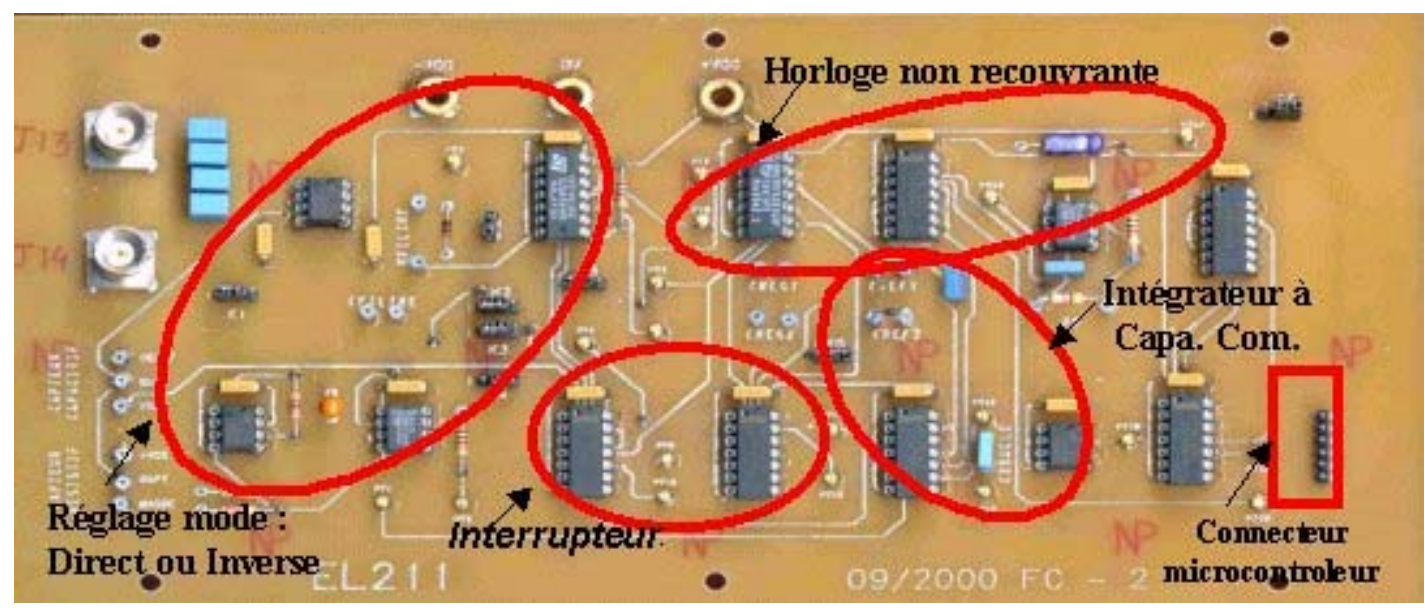

Fig. 5. Vue de dessus du circuit imprimé (Carte modulateur Sigma-Delta).

Certaines cartes ont été mises à disposition des élèves qui ont ainsi pu de manière autonome corréler le didacticiel avec des mesures effectuées sur la carte. Une pédagogie du type inductif a aussi été utilisée autour de cette carte pour laisser les élèves découvrir au travers des TP les notions de capacités commutées et ensuite aller chercher dans le didacticiel les formulations théoriques. 
De plus, cette carte peut être entièrement pilotée par microcontrôleur pour réaliser un mésosystème intelligent adaptable à la numérisation des signaux issus de plusieurs sortes de cellules sensibles (type capacitif, résistif, résistif polarisé). Cette commande «intelligente » permet alors d'ajouter des fonctionnalités à la chaîne de mesure :

- $\quad$ auto test ;

- changement de mode pour éliminer les effets de l'offset et de l'injection de charge due aux interrupteurs ;

- filtrage numérique (plusieurs algorithmes peuvent être programmés), pour transformer le modulateur Sigma Delta en convertisseur Sigma Delta ;

- $\quad$ mise en réseau de plusieurs cartes.

Au moins trois autres séances de travaux pratiques sont nécessaires pour mettre en valeur toutes ces nouvelles fonctionnalités. C'est alors une phase de traitement numérique des données qui peut être exploitée via la programmation d'un microcontrôleur.

\section{Conclusion}

L'utilisation d'un didacticiel appliqué à l'électronique des microsystèmes a permis d'améliorer, chez les étudiants, la compréhension des principes physiques mis en jeu et la capacité d'apprentissage en mode autonome. En l'associant à une maquette pédagogique, les élèves ont pu rester en contact avec la pratique et ainsi confronter en direct la théorie avec des relevés de mesure.

Une démonstration du didacticiel comme de la carte est prévue au cours de la présentation.

\section{Références bibliographiques}

[1] F. Baillieu et Y. Blanchard, Signal analogique et capacités commutées (Dunod, 1994).

[2] F. Baillieu, Y. Blanchard, P. Loumeau, H. Petit et J. Porte,Capacités commutées et applications (Dunod, 1996).

[2] F. Baillieu et O. Vancauwenberghe, Chapitre 1 in Du composant élémentaire au système - Mesure et Instrumentation 2 (Hermès, 2000). 